\title{
Effects of screening and brief intervention training on resident and faculty alcohol intervention behaviours: a pre- post-intervention
} assessment

\author{
J Paul Seale ${ }^{* 1}$, Sylvia Shellenberger ${ }^{1}$, John M Boltri ${ }^{1}$, IS Okosun ${ }^{2}$ and \\ Barbara Barton ${ }^{1}$
}

Address: ${ }^{1}$ Department of Family Medicine, Mercer University School of Medicine and Medical Center of Central Georgia, 3780 Eisenhower Parkway, Macon GA 31210, USA and 'Institute of Public Health, Georgia State University, One Park Place South, Sixth Floor, Suite 660, Atlanta, GA 30302, USA

Email: J Paul Seale* - seale.paul@mccg.org; Sylvia Shellenberger - shellenberger.sylvia@mccg.org; John M Boltri - boltri.john@mccg.org; IS Okosun - iokosun@gsu.edu; Barbara Barton - barton.barbara@mccg.edu

* Corresponding author

Published: 4 November 2005

BMC Family Practice 2005, 6:46 doi:10.1 186/147|-2296-6-46
Received: 10 June 2005

Accepted: 4 November 2005

This article is available from: http://www.biomedcentral.com/I47I-2296/6/46

(c) 2005 Seale et al; licensee BioMed Central Ltd.

This is an Open Access article distributed under the terms of the Creative Commons Attribution License (http://creativecommons.org/licenses/by/2.0), which permits unrestricted use, distribution, and reproduction in any medium, provided the original work is properly cited.

\begin{abstract}
Background: Many hazardous and harmful drinkers do not receive clinician advice to reduce their drinking. Previous studies suggest under-detection and clinician reluctance to intervene despite awareness of problem drinking (PD). The Healthy Habits Project previously reported chart review data documenting increased screening and intervention with hazardous and harmful drinkers after training clinicians and implementing routine screening. This report describes the impact of the Healthy Habits training program on clinicians' rates of identification of PD, level of certainty in identifying PD and the proportion of patients given advice to reduce alcohol use, based on self-report data using clinician exit questionnaires.

Methods: 28 residents and 10 faculty in a family medicine residency clinic completed four cycles of clinician exit interview questionnaires before and after screening and intervention training. Rates of identifying PD, level of diagnostic certainty, and frequency of advice to reduce drinking were compared across intervention status (pre vs. post). Findings were compared with rates of PD and advice to reduce drinking documented on chart review.

Results: I,052 clinician exit questionnaires were collected. There were no significant differences in rates of PD identified before and after intervention $(9.8 \%$ vs. $7.4 \%, p=.308)$. Faculty demonstrated greater certainty in PD diagnoses than residents $(p=.028)$ and gave more advice to reduce drinking $(p=.042)$ throughout the program. Faculty and residents reported higher levels of diagnostic certainty after training $(p=.039$ and .030 , respectively). After training, residents showed greater increases than faculty in the percentage of patients given advice to reduce drinking $(p=.038)$, and patients felt to be problem drinkers were significantly more likely to receive advice to reduce drinking by all clinicians $(50 \%$ vs. $75 \%, p=.047)$. The number of patients receiving advice to reduce drinking after program implementation exceeded the number of patients felt to be problem drinkers. Recognition rates of PD were four to eight times higher than rates documented on chart review $(P=.028)$.

Conclusion: This program resulted in greater clinician certainty in diagnosing PD and increases in the number of patients with PD who received advice to reduce drinking. Future programs should include booster training sessions and emphasize documentation of PD and brief intervention.
\end{abstract}




\section{Background}

Patients with hazardous and harmful drinking patterns are commonly encountered by primary care clinicians worldwide. Studies from the U.S., Europe and Australia indicate that $10-40 \%$ of patients seen in primary care settings engage in hazardous or harmful drinking [1-5]. This group of patients, sometimes referred to as "risky drinkers," includes patients who meet diagnostic criteria for alcohol abuse and alcohol dependence, as well as patients who exceed recommended "safe drinking guidelines" of the National Institute for Alcohol Abuse and Alcoholism and are at increased risk for alcohol-related problems [2]. Numerous randomized controlled trials have demonstrated that screening and brief intervention (SBI) are effective in reducing alcohol consumption among such drinkers [6-8], yet SBI still remains underutilized in primary care practices. Several older studies, many of them based on chart review data, suggested that problem drinking (PD) was largely undetected and untreated in primary care [9-12]. Two recent studies using other measurement techniques (clinician exit questionnaires and direct observation) have indicated that discussions of alcohol use by clinicians and patients in primary care are more frequent than previously thought, occurring in $9-10 \%$ of primary care encounters $[13,14]$. Nonetheless, studies conducted in the U.S., Australia, the United Kingdom and Finland indicate that clinicians frequently fail to screen for PD, and fail to address PD in at least one-third to one-half of cases, even when the diagnosis is known [13,15-20]. Indeed, 72\% of U.S. primary physicians surveyed in 1999 reported that they preferred not to counsel early problem drinkers themselves but rather to refer them to a nurse trained in behavioural interventions [21]. Residents are less likely to perform brief interventions than faculty physicians [22], and only $13-20 \%$ of problem drinkers report receiving advice to reduce drinking, a key element of most effective SBI programs $[17,22,23]$. While studies have demonstrated that providing experiential training can increase primary care clinicians' rates of providing brief advice to problem drinkers [24-27], studies of the effect of resident training have yielded mixed results $[28,29]$. We previously reported initial findings from the Healthy Habits Project, a training program designed to increase SBI rates in a family medicine residency program using a combination of clinician training and clinic systems intervention. Based primarily on chart review, findings indicated that following program implementation, alcohol interventions increased from $12.5 \%$ to $47.7 \%$ of patients who screened positive for hazardous and harmful drinking, and that clinicians who were prompted with positive screening results gave advice to reduce drinking to $72 \%$ of patients [30]. This report provides further information, obtained using clinician exit questionnaires, regarding the impact of this SBI training program on resident and faculty physician alcohol intervention attitudes and behav- iours. We hypothesized that the SBI training program would result in the following changes for both resident and faculty clinicians: (1) greater recognition of PD, (2) increased certainty in identifying PD, and (3) increased advice to reduce drinking.

\section{Methods}

The Healthy Habits Project utilized a combination of clinician training and a clinic-wide systems intervention program to increase alcohol screening and brief intervention in a family practice residency clinic in the southeastern U.S. Details of the program's systems interventions and training procedures have been previously described [30]. Briefly, the clinic is staffed by residents (28 physicians completing three years of post medical school training) and by faculty ( 8 family physicians and 2 physician assistants). All of the clinic's clinicians (residents and faculty) participated and gave written consent, and efforts were made to screen all adult patients during a 12-month period. The study was approved by the Institutional Review Board of the Medical Center of Central Georgia. Alcohol screening and intervention procedures were modeled after the University of Connecticut's Cutting Back Screening and Brief Intervention Program [26,31]. A clinic team implemented alcohol screening using the threequestion AUDIT-C, a validated screening instrument for hazardous and harmful drinking [32-34], embedded in a health questionnaire distributed by registration clerks. After scoring the AUDIT-C, nurses asked screen-positive patients to complete the ten-question Alcohol Use Disorders Identification Test (AUDIT) [35]. All clinicians underwent 3 hours of training in which they were instructed to score the 10-question AUDIT and conduct brochure-guided brief interventions with all screen-positive patients. A key component of the intervention was negotiating a clinician-patient contract to reduce alcohol consumption during the ensuing 30 days. Training included a lecture, demonstration interviews, and roleplaying exercises. Clinicians were asked to reschedule a follow-up visit within 30 days. Regular feedback sessions with clinic staff, nurses, and clinicians were implemented to encourage compliance with protocols. Periodic program evaluation by the project's implementation committee resulted in minor modifications of protocols which created three separate implementation phases: A (Months 1-3), B (Months 4-6), and C (Months 7-12). During Phase $B$, in an attempt to boost overall patient screening rates, monthly feedback sessions were scheduled with clinic staff and nurses. During Phase C, as a further attempt to increase screening rates, alcohol screening questions were integrated into the clinic's mandatory annual clinical information update. 
Table I: Baseline comparability: demographics of residents and faculty

\begin{tabular}{llll}
\hline Variable & Faculty $(\mathbf{n}=\mathbf{~ 1 0 )}$ & Residents $(\mathbf{n}=\mathbf{2 8})$ & Total Participants $(\mathbf{n}=\mathbf{3 8})$ \\
\hline $\begin{array}{l}\text { Age, years mean (SD) } \\
\text { \% Female }\end{array}$ & $44.2(\mathrm{SD} 6.4)$ & $34.0(\mathrm{SD} 6.6)$ & 36.7 (SD 7.9) \\
\hline
\end{tabular}

\section{Assessments \\ Clinician exit questionnaires (CEQ's)}

A pre-post research design was used to measure the project's impact on clinicians' level of certainty in identifying problem drinking and their self-report of advice given to patients to reduce their drinking. Four sets of CEQ's were collected (longitudinally during the baseline month and Implementation Phase A, then for 1-2 weeks at the end of Phases $\mathrm{B}$ and $\mathrm{C}$ ). Clinicians were asked to complete a three-question instrument (see Appendix 1) in which they reported whether they thought the patient they had just seen had a drinking problem, their degree of certainty of this diagnosis on a five-point Likert-type scale, and whether they had suggested that the patient stop drinking or cut back. All CEQ's were completed at the conclusion of individual patient encounters. The first, second, and fourth collections were performed by a research assistant, who approached individual clinicians in the hall immediately following two patient visits during each halfday clinic session. During the third collection period (Phase B), exit questionnaires were attached to each patient's routing form, and clinicians were requested to complete them on each patient seen.

\section{Chart reviews}

During two one-month periods (at baseline and during the project's final month), chart reviews of every fourth adult patient seen were conducted by one of two investigators (JPS, BB), who reviewed clinic notes from the patient's database, problem list, index visit, and all office visits during the previous year. Patients were considered "diagnosed" if alcohol abuse, alcohol dependence, heavy drinking, or similar terms were listed in the assessment area of a clinic note or the patient's problem list. Documentation of advice to quit drinking, cut back, or attend formal alcohol treatment was considered "intervention."

\section{Statistical analysis}

Statistical programs available in SPSS for Windows were used for analysis [36]. Pre- and post-assessments of the clinician's diagnostic impression, degree of certainty, and advice to decrease drinking were compared. Data were analyzed separately for faculty and residents, and then for all clinicians. Data from the three implementation phases of the study were analyzed separately and as aggregate data. Additional analyses were performed after removing CEQ's completed by two of the study's co-investigators
(JPS, JB) to look for possible bias. All data were analyzed using Pearson's Chi-squared test. For the tables with expected cell frequencies less than five, Fisher's exact test was employed. Chi-square test for trend was used to determine diagnostic changes over time for faculty and residents. Analysis of variance (ANOVA) was used to compare differences between faculty and residents across baseline and study phases for the study's three primary outcome measures - mean number of subjects recognized with problem drinking, level of certainty in recognition, and number of patients given advice to reduce drinking. ANOVA was also used to assess possible impact of clinician gender and age on these three outcome measures. Test of linear trends was used to assess whether clinical diagnoses of problem drinking increased or decreased over time for patients who were evaluated by faculty, residents and all clinicians. The customary p-values of $<0.05$ were used to indicate statistical significance.

\section{Results}

Thirty-eight clinicians completed exit questionnaires from a total of 1,052 patient encounters (164 at baseline, 888 during the implementation period). Residents were significantly younger than faculty clinicians (mean age 34 vs. 44 years, $\mathrm{p}<.0001$; see Table 1$)$. Although a higher percentage of residents were female, when compared to faculty, (64\% vs. $30 \%)$, differences were not statistically significant $(\mathrm{p}=.078)$. During the third data collection period, in which exit questionnaires were self-administered, questionnaires were completed on $44 \%$ of adult patients seen during a one-week period. Chart reviews were conducted on 178 charts from the one-month baseline period and 200 charts from Month 12 of implementation.

\section{Recognition of problem drinking}

Overall, there was no statistically significant difference in mean number of subjects recognized as problem drinkers by faculty and residents across baseline and study phases. Clinicians reported problem drinking in $9.8 \%(16 / 164)$ of patients at baseline and 7.4\% (66/888) of patients during the project's three implementation phases $(\mathrm{p}=.308)$. These rates are similar to the rates of risky drinking obtained by questionnaire screening using the AUDIT-C (8.6\% during the baseline period and 8.0\% during the implementation period [30]). Faculty impressions of problem drinking remained relatively stable throughout the project, while residents' diagnostic impressions 
Table 2: Changes in numbers and percent of patients diagnosed as problem drinkers across study phases

\begin{tabular}{llll}
\hline & $\begin{array}{l}\text { Diagnoses by faculty } \\
\text { N (\%) }\end{array}$ & $\begin{array}{l}\text { Diagnoses by residents } \\
\text { N (\%) }\end{array}$ & $\begin{array}{l}\text { Diagnoses by all clinicians } \\
\text { N (\%) }\end{array}$ \\
\hline $\begin{array}{l}\text { Study Phase } \\
\text { Baseline }\end{array}$ & & & $164(9.8)$ \\
Phase A & $77(9.1)$ & $86(10.5)$ & $501(8.4)$ \\
Phase B & $222(9.5)$ & $279(7.5)$ & $256(6.3)$ \\
Phase C & $141(5.7)$ & $90(6.7)$ & $131(6.1)$ \\
p-value & $78(10.2)$ & $43(0)$ & .148 \\
\hline
\end{tabular}

$\mathrm{P}$-value is for linear trends

showed a trend toward decline over time (see Table 2; $\mathrm{p}=$ .052 ). Despite equivalent percentages of patients who screened positive for hazardous drinking using the AUDIT-C at baseline (8.6\%) and during the final phase of the study $(8.8 \%)$, recognition rates for PD measured by CEQ were significantly higher than rates documented in patient charts during both periods (baseline: $9.8 \%$ vs. $1.2 \%, \mathrm{p}=.035$, and final study phase: $6.1 \%$ vs. $1.5 \%, \mathrm{p}=$ $.008)$.

\section{Certainty in identifying problem drinking}

Clinicians' mean level of certainty regarding the presence or absence of hazardous or harmful drinking among all patients was high both before $(4.23,+/-0.98)$ and after SBI implementation $(4.21,+/-0.97)$. ANOVA showed no differences between certainty levels for faculty and residents across all patient encounters $(p=.446)$. Differences were observed, however, when analyses were limited to patients felt to have a drinking problem. Faculty level of certainty in patients with PD was greater than residents' level of certainty both before SBI program implementation (4.14 vs. 3.56$)$ and after implementation (4.38 vs. $3.96), p=.028$. After program implementation, levels of certainty for patients with PD increased for both faculty ( 4.38 vs. $4.14, \mathrm{p}=.039)$ and residents (3.96 vs. $3.56, \mathrm{p}=$ $.030)$.

\section{Advice to reduce drinking}

Overall, comparative analysis of mean number of patients given advice to reduce drinking by faculty and residents across baseline and study phases did not demonstrate statistically significant changes. CEQ responses indicated that clinicians gave brief advice to $6.1 \%(10 / 164)$ of all patients seen at baseline and $8.6 \%(75 / 874)$ of patients seen during the project's implementation phase $(\mathrm{p}=$ .287); see Figure 1. Analyses revealed no impact of gender or clinician age on clinician advice rates. Intervention rates were highest during Phase A, the first three months following training. Rates decreased modestly for all providers during Phase $\mathrm{B}$, then in Phase $\mathrm{C}$ showed declines for residents and increases for faculty. Comparisons of the mean number of all subjects receiving advice to reduce drinking by faculty vs. residents across study phases found that faculty were significantly more likely to advise patients to reduce drinking $(\mathrm{p}=.042)$. When baseline brief advice rates are compared with those during the combined intervention periods, residents showed greater increases in brief advice rates (from $4.7 \%$ to $7.8 \%$ ) than faculty (from $7.8 \%$ to $9.3 \%$ ); $\mathrm{p}=.041$. Intervention rates increased among faculty who were co-investigators in the study $(11.5 \%$ vs. $16.7 \%, \mathrm{p}=.049)$ but showed no significant change among faculty who were not $(9.8 \%$ vs. $7.0 \%$, $\mathrm{p}=.421$ ). When the analysis was limited to patients felt by clinicians to be problem drinkers, advice to reduce drinking increased from $50 \%(8 / 16)$ of problem drinkers during the baseline phase to $75 \%(49 / 65)$ during the three implementation phases $(\mathrm{p}=.038)$. While the overall number of patients receiving advice to reduce drinking was less than the number of patients thought to be problem drinkers during the baseline period $(10 / 16$, or $62 \%)$, the number of patients receiving advice to reduce drinking during the implementation phase actually exceeded the number of patients thought to be problem drinkers (75/ 66 , or $114 \%$ ).

\section{Effect of clinician age and gender}

Finally, ANOVA showed no statistically significant effect of age or gender of the faculty and residents on the study's three primary outcome measures - mean number of subjects recognized with problem drinking, level of certainty in recognition, and number of patients given advice to reduce drinking.

\section{Discussion \\ Clinician attitudes and behaviours related to brief intervention}

To our knowledge, this study is the third residency-based SBI training program to demonstrate positive changes in clinician attitudes and behaviours related to alcohol intervention. Researchers at the University of Massachusetts previously reported increases in readiness to intervene and in actual performance of brief interventions performed by residents and faculty physicians in a similar program which also provided clinician training, routine 


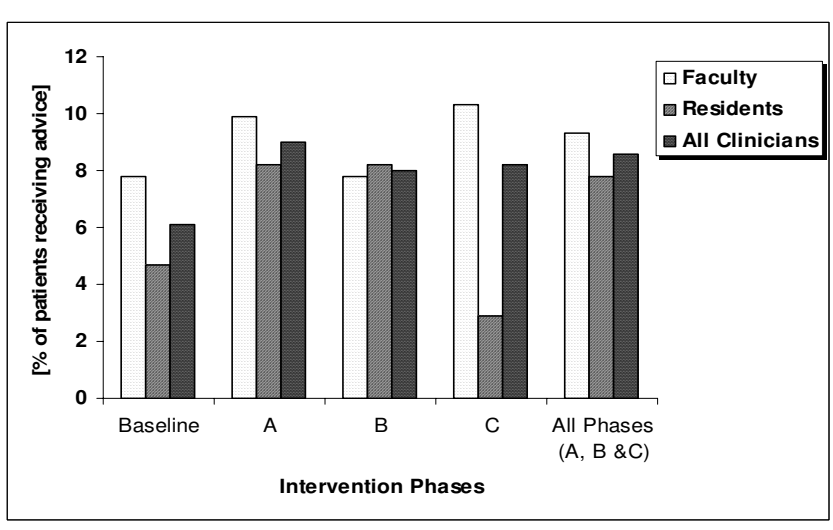

Figure I

Changes in clinician advice to reduce drinking (all patients).

screening, and clinician prompting with screen-positive patients [24,37]. Wilk and Jensen [28] reported increases in brief interventions by residents in interviews with unannounced standardized patients following brief intervention training. In our study, both faculty and residents showed significant increases in their certainty in diagnosing PD after training. Residents showed greater increases in diagnostic certainty and intervention rates than faculty, an important finding in light of evidence from our study and others [22] that residents are less likely than faculty to perform brief interventions. Because previous studies have demonstrated that not all alcohol-related discussions include advice to reduce drinking, a key element of SBI cited by the US Preventive Services Task Force [38], our evaluation was focused on the percentage of patients who actually received advice to reduce drinking. During the project's intervention phase, clinicians reported a modest increase in providing advice to reduce drinking (from $6.8 \%$ to $8.6 \%$ ). While this increase did not reach statistical significance $(\mathrm{p}=.287)$, a significant increase was seen in the percent of perceived problem drinkers receiving such advice ( $50 \%$ to $75 \%, \mathrm{p}=.047$ ). These findings, although based on small numbers of encounters with problem drinkers, are consistent with previous studies indicating that clinicians who have received SBI training are more confident in their ability to conduct brief interventions and more likely to intervene with problem drinkers $[26,39,40]$. In contrast to some earlier studies which found younger clinicians to be more willing to intervene than older clinicians $[39,41,42]$, this study found no impact of age or gender on confidence in diagnoses of problem drinking or advice given to reduce drinking. Reasons for this finding are unclear, but could be related to the relatively young age of the overall group (mean age 37, with only two clinicians over age 50) or to the fact that clinicians of all ages received intensive training, which has been shown to correlate with greater clini- cian confidence and performance levels $[40,42,43]$. Further research is needed to determine which of the training program's multiple components - experiential training, implementation of routine alcohol screening performed by nurses, prompting clinicians with positive screening results and assessment data, or compliance feedback regarding intervention rates - were most critical in achieving increased intervention rates.

Overall, our study indicated that faculty prescribed reduction in drinking to more patients than residents did, but that resident intervention rates showed greater increases after training than faculty rates. Resident interventions did, however, show a non-significant trend toward decline toward the end of the one-year study. While this could represent a loss of training effect over time, evaluation results also may have been confounded by conducting the final exit interview evaluation in July, when skilled third-year residents had just graduated and newly-promoted residents were struggling to manage increased patient volumes. Interestingly, intervention prompt forms reviewed for the previous report on this project [30] indicate that there was no decline in resident interventions when prompted with positive screening results during this period: brief interventions were performed in 75\% (6/8) of cases. Regardless of the reason for the decline, findings suggest that reinforcement methods such as booster sessions are needed to maintain behaviours taught in the initial training sessions.

One of this study's most encouraging findings is the fact that the number of patients receiving advice to reduce drinking after SBI training actually exceeded the number of patients felt to be problem drinkers. This finding suggests that the SBI program was successful in legitimizing and normalizing conversations about alcohol, such that clinicians felt more at ease in addressing alcohol use in a variety of clinical scenarios, and indicates that at-risk drinkers as well as problem drinkers received brief advice to reduce their drinking.

\section{Recognition of problem drinking}

In contrast to our expectation, clinician exit questionnaires did not reflect increased recognition of problem drinking after program implementation. While this could be due to the relatively high levels of clinician recognition at baseline, it could also reflect decreased clinician vigilance once routine screening protocols were in place. This finding, if confirmed in other studies, has implications for future SBI training programs, especially in light of the fact that most "routine" screening systems are not effective in detecting all risky drinkers. Clinician training should include reminders that significant numbers of risky drinkers may remain unscreened or escape detection via questionnaire screening, whose sensitivity rarely exceeds $85 \%$, 
and clinicians must remain alert to clinical clues related to hazardous or problem drinking.

\section{Documentation of problem drinking}

One striking finding of this study is the marked difference between the perceived level of problem drinking (7.4$9.8 \%$ of patients) and the documentation of such diagnoses in the medical record $(1.2-1.5 \%)$, suggesting that clinicians document perceived problem drinking in less than $25 \%$ of cases. Significant underdocumentation of alcohol disorders has been noted in several other previous studies $[15,44,45]$, pointing out an important methodological flaw in many previous studies which have presumed that problem drinkers are "undiagnosed" based on chart review data [9-12]. This finding suggests that future studies assessing clinician recognition and intervention rates should utilize more sensitive measures such as clinician or patient exit interviews or direct observation, and that future training efforts should address documentation of alcohol disorders in the medical record.

\section{Limitations of this pilot study}

It is important to take into account possible methodological limitations of this pilot study. First, the sample size was not large, particularly during the baseline assessment period. However, the numbers in each group were sufficient to detect between-group differences of at least 10\% or greater in recognition of problem drinking and at least $10 \%$ or greater in advice to reduce drinking. Secondly, a change in methodology during the study's second implementation phase could have confounded study results. During this period, exit questionnaires were attached to each patient's routing form, and clinicians were requested to complete them on each patient seen. The return rate was low (44\%), and selection bias may have occurred, perhaps in favour of patients whom clinicians identified as problem drinkers or in favour of patients who received an intervention. Nonetheless, both clinicians' recognition (6.7\% of patients) and intervention rates $(8.0 \%)$ during this phase are within the range of the other phases of the study and do not suggest selection bias. Thirdly, this study lacked a criterion diagnosis for confirming problem drinking in patients considered by clinicians to be problem drinkers. While some patients may have been incorrectly diagnosed, rates of problem drinking recognition by clinicians throughout this study are similar to this study's previously-published problem drinking estimates obtained by AUDIT-C questionnaire screening, and only slightly lower than the estimated U.S. problem drinking prevalence of $11 \%$ in primary care [1]. Future studies comparing clinician's impressions with the results of standardized diagnostic interviews for problem drinking could help to clarify this issue.

\section{Conclusion}

This SBI training program resulted in greater clinician certainty in diagnosing PD and modest but significant increases in the number of patients with PD who received advice to reduce their drinking. The program shows promise for helping translate SBI findings into residency and clinical practice. Trends toward lower rates of identification of PD and intervention by residents during the program's later phases suggest a need for booster training sessions. Increased emphasis on documentation of problem drinking and brief intervention is also needed.

\section{Appendix I: exit questionnaire for clinicians}

1 ). Do you think this patient has a drinking problem? Yes__ No

\section{$2)$. What is your degree of certainty?}

\section{$\begin{array}{llllll}\text { Uncertain } 1 & 2 & 3 & 4 & 5 & \text { Certain }\end{array}$}

3. Did you talk with this patient today about cutting back or quitting?
No Yes

\section{Competing interests}

The author(s) declare that they have no competing interests.

\section{Authors' contributions}

JPS conceived of and designed the study, supervised acquisition of the data, participated in interpretation of data and drafted the manuscript. SS assisted in designing and implementing the study, interpreting the data, and editing the manuscript. JMB assisted in designing and implementing the study, interpreting the data and editing of the manuscript. ISO performed the statistical analysis and participated in drafting and editing the manuscript. BB participated in data collection, study design and implementation, and data analysis.

\section{Acknowledgements}

The authors would like to thank Dr. Dan Vinson for his help in study design and the directors of the Cutting Back program for sharing their technical expertise and the materials used in their program. We also thank Dr. Piyush Patel for his assistance in data collection. Financial support for this project was provided by the Clinical Research Center of the Medical Center of Central Georgia and by Department of Health and Human Services Grant \#I DI2 HP 00159.

\section{References}

I. Fleming MF, Graham AW: Screening and brief interventions for alcohol use disorders in managed care settings. Recent Dev Alcohol 2001 , I5:393-416.

2. National Institute on Alcohol Abuse and Alcoholism: Helping Patients with Alcohol Problems: A Health Practitioner's Guide. National Institutes of Health, Public Health Service, U. S. 
Department of Health and Human Services; 2003. NIH Publication No. 03-03769

3. Saitz R: Clinical practice. Unhealthy alcohol use. N Engl J Med 2005, 352:596-607.

4. World Health Organization: Alcohol consumption in the European Region - consumption, harm and policies. Copenhagen, World Health Organization Regional Office for Europe; 2001.

5. Richmond RL, G-Novak K, Kehoe L, Calfas G, Mendelsohn COP, Wodak $A$ : Effect of training on general practitioners' use of a brief intervention for excessive drinkers. Aust N ZJ Public Health 1998, 22:206-9.

6. Moyer A, Finney JW, Swearingen CE, Vergun P: Brief interventions for alcohol problems: A meta-analytic review of controlled investigations in treatment-seeking and non-treatmentseeking populations. Addiction 2002, 97:279-292.

7. Fleming $M$, Manwell LB: Brief intervention in primary care settings. A primary treatment method for at-risk, problem, and dependent drinkers. Alcohol Res Health 1999, 23: | 28-137.

8. Bertholet N, Daeppen JB, Wietlisbach V, Fleming M, Burnand B: Reduction of alcohol consumption by brief alcohol intervention in primary care: systematic review and meta-analysis. Arch Intern Med 2005, 165:986-95.

9. Moore RD, Malitz FE: Underdiagnosis of alcoholism by residents in an ambulatory medical practice. J Med Educ 1986, 6I:46-52.

10. Woodall HE: Alcoholics remaining anonymous: resident diagnosis of alcoholism in a family practice center. J Fam Pract 1988, 26:293-6.

II. Curtis JR, Geller G, Stokes EJ, Levine DM, Moore RD: Characteristics, diagnosis, and treatment of alcoholism in elderly patients. J Am Geriatr Soc 1989, 37:3 10-6.

12. Cerise FP, Scarinci IC, Thibodaux R, Cannatella M, Stark S, Brantley $\mathrm{PJ}$ : Alcoholism among indigent inpatients: identification and intervention by internal medicine residents. South Med J 1998, 91:27-32.

13. Vinson DC, Elder N, Werner JJ, Vorel LA, Nutting PA: Alcoholrelated discussions in primary care: $A$ report from ASPN. Fam Pract 2000, 49:28-33.

14. Stange KC, Zyzanski SJ, jaen CR, Callahan EJ, Kelly RB, Gillanders WR, Shank JC, Chao J, Medalie JH, Miller WL, Crabtree BF, Flocke SA, Gilchrist VJ, Langa DM, Goodwin MA: Illuminating the 'black box'. A description of $\mathbf{4 4 5 4}$ patient visits to $\mathbf{1 3 8}$ family physicians. J Fam Pract 1998, 46:377-89.

15. Conigliaro J, Lofgren RP, Hanusa BH: Screening for problem drinking: impact on physician behavior and patient drinking habits. J Gen Intern Med 1998, 13:25I-6.

16. Kaner EF, Heather N, Brodie J, Lock CA, McAvoy BR: Patient and practitioner characteristics predict brief alcohol intervention in primary care. Br J Gen Pract 200I, 5 I:822-827.

17. Aalto M, Pekuri P, Seppa K: Primary health care professionals activity in intervening in patients' alcohol drinking during a 3-year brief intervention implementation project. Drug Alcohol Depend 2003, 69:9-14.

18. Rydon P, Redman S, Sanson-Fisher RW, Reid AL: Detection of alcohol-related problems in general practice. J Stud Alcohol 1992 53:197-202.

19. Richmond RL, Anderson P: Research in general practice for smokers and excessive drinkers in Australia and the UK. III. Dissemination of interventions. Addiction 1994, 89:49-62.

20. Kaner EF, Lock CA, McAvoy BR, Heather N, Gilvarry E: A RCT of three training and support strategies to encourage implementation of screening and brief alcohol intervention by general practitioners. BrJ Gen Pract 1999, 49:699-703.

21. Spandorfer JM, Israel Y, Turner B]: Primary care physicians' views on screening and management of alcohol abuse: inconsistencies with national guidelines. J Fam Pract 1999, 48:899-902.

22. Bradley KA, Epler AJ, Bush KR, Sporleder IL, Dunn CW, Cochran NE, Braddock $\mathrm{CH}$ 3rd, McDonell MB, Fihn SD: Alcohol-related discussions during general medicine appointments of male VA patients who screen positive for at-risk drinking. I Gen Intern Med 2002, 17:315-26.

23. Aalto M, Pekuri P, Seppa K: Primary health care professionals activity in intervening in patients' alcohol drinking: a patient perspective. Drug Alcohol Depend 2002, 66:39-43.

24. Adams A, Ockene J, Wheeler E, Hurley T: Alcohol counseling: Physicians will do it. J Gen Intern Med 1998, 13:692-698.
25. Richmond RL, G-Novak K, Kehoe L, Calfas G, Mendelsohn CP, Wodak $A$ : Effect of training on general practitioners' use of a brief intervention for excessive drinkers. Aust N Z J Public Health 1998, 22:206-9.

26. Babor TF, Higgins-Biddle JC, Higgins PS, Gassman RA, Gould BE: Training medical providers to conduct alcohol screening and brief interventions. Subst Abus 2004, 25:17-26.

27. Roche AM, Stubbs JM, Sanson-Risher RW, Saunders J: A controlled trial of educational strategies to teach medical students brief intervention skills for alcohol problems. Prev Med 1997 26:78-85

28. Wilk $\mathrm{Al}$, Jensen NM: Investigation of a brief teaching encounter using standardized patients: teaching residents alcohol screening and intervention. J Gen Intern Med 2002, 17:356-60.

29. Saitz R, Horton NJ, Sullivan LM, Moskowitz MA, Samet JH: Addressing alcohol problems in primary care: a cluster randomized, controlled trial of a systems intervention. The screening and intervention in primary care (SIP) study. Ann Intern Med 2003, 138:372-82

30. Seale JP, Shellenberger S, Tillery WL, Boltri J, Vogel R, Barton B, McCauley M: Implementing Alcohol Screening \& Intervention in a Family Medicine Residency Clinic. Substance Abuse 2005 in press.

31. Babor TF, Higgins-Biddle J, Dauser D, Higgins P, Burleson J: Alcohol screening and brief intervention in primary care settings: implementation models and predictors. I Stud Alcohol 2005 , 66:36I-8.

32. Bush K, Kivlahan DR, McDonnell MB, Finn SD, Bradley KA: The AUDIT alcohol consumption questions (AUDIT-C): an effective brief screening test for problem drinking. Ambulatory Care Quality Improvement Project (ACQUIP). Alcohol Use Disorders Identification Test. Arch Intern Med 1998, I 58:1789-1795

33. Gordon AJ, Maisto SA, McNeil M, Kraemer KL, Conigliaro R, Kelly $\mathrm{ME}$, Conigliaro J: Three questions can detect hazardous drinkers. J Fam Pract 200I, 50:313-320.

34. Bradley KA, Bush KR, Epler AJ, Dobie DJ, Davis TM, Sporleder JL, Maynard C, Burman ML, Kivlahan DR: Two brief alcohol-screening tests from the Alcohol Use Disorders Identification Test (AUDIT): Validation in a female Veterans Affairs patient population. Arch Intern Med 2003, 163:821-829.

35. Saunders JB, Aasland OG, Babor TF, de la Fuente JR, Grant M: Development of the Alcohol Use Disorders Identification Test (AUDIT): WHO collaborative project on early detection of persons with harmful alcohol consumption - II. Addiction 1993, 88:79I-804.

36. SPSS for Windows Release II.5.0 [computer program]. Chicago: SPSS; 2002

37. Ockene JK, Wheeler EV, Adams A, Hurley TG, Hebert J: Provider training for patient-centered alcohol counseling in a primary care setting. Arch Intern Med 1997, 157:2334-4I.

38. Whitlock EP, Polen MR, Green CA, Orleans T, Klein J, U.S. Preventive Services Task Force: Behavioral counseling interventions in primary care to reduce risky/harmful alcohol use by adults: a summary of the evidence for the U.S. Preventive Services Task Force. Ann Intern Med 2004, 140:557-68.

39. Clement $\mathrm{S}$ : The identification of alcohol-related problems by general practitioners. Br J Addict 1986, 81:257-64

40. Kaner EF, Wutzke S, Saunders JB, Powell A, Morawski J, Bouix JC WHO Brief Intervention Study Group: Impact of alcohol education and training on general practitioners' diagnostic and management skills: findings from a World Health Organization collaborative study. J Stud Alcohol 200I, 62:62I-7.

4I. Roche AM, Richard GP: Doctors' willingness to intervene in patients' drug and alcohol problems. Soc Sci Med I99|, 33:|053-6|

42. Roche AM: Increasing primary care providers' willingness to intervene in alcohol- and drug-related problems: A review. Substance Abuse 1996, 1 7:201-217.

43. Fucito L, Gomes B, Murnion B, Haber P: General practitioners' diagnostic skills and referral practices in managing patients with drug and alcohol-related health problems: implications for medical training and education programmes. Drug Alcohol Rev 2003, 22:417-24. 
44. McDonald AJ 3rd, Wang N, Camargo CA Jr: US emergency department visits for alcohol-related diseases and injuries between 1992 and 2000. Arch Intern Med 2004, 164:531-7.

45. Seppa K, Lahtinen T, Antila S, Aalto M: Alcohol drinking among emergency patients - alcometer use and documentation. Alcohol Alcohol 2004, 39:262-5.

\section{Pre-publication history}

The pre-publication history for this paper can be accessed here:

http://www.biomedcentral.com/1471-2296/6/46/prepub

Publish with Bio Med Central and every scientist can read your work free of charge

"BioMed Central will be the most significant development for disseminating the results of biomedical research in our lifetime. " Sir Paul Nurse, Cancer Research UK

Your research papers will be:

- available free of charge to the entire biomedical community

- peer reviewed and published immediately upon acceptance

- cited in PubMed and archived on PubMed Central

- yours - you keep the copyright

Submit your manuscript here:

http://www.biomedcentral.com/info/publishing_adv.asp 